\title{
DÜBLIN
}

Technological University Dublin

ARROW@TU Dublin

2009-01-01

\section{A Single Beam Data Writing Process for Holographic Data Storage}

\author{
Suzanne Martin \\ Technological University Dublin, suzanne.martin@tudublin.ie \\ Denis Bade \\ Technological University Dublin, denis.bade@tudublin.ie \\ Izabela Naydenova \\ Technological University Dublin, izabela.naydenova@tudublin.ie
}

See next page for additional authors

Follow this and additional works at: https://arrow.tudublin.ie/cieocon2

Part of the Optics Commons

\section{Recommended Citation}

Martin, S., Bade, D. \& Naydenova, I. (2009). A single beam data writing process for holographic data storage. Optical Data Storage Topical Meeting, ODS '09., pg.70-72, 10-13 May. doi: 10.1109/

ODS.2009.5031757

This Conference Paper is brought to you for free and open access by the Centre for Industrial and Engineering Optics at ARROW@TU Dublin. It has been accepted for inclusion in Conference Papers by an authorized administrator of ARROW@TU Dublin. For more information, please contact arrow.admin@tudublin.ie, aisling.coyne@tudublin.ie,gerard.connolly@tudublin.ie. Funder: Science Foundation Ireland Technological Sector Research Strand 1 
Authors

Suzanne Martin, Denis Bade, Izabela Naydenova, and Vincent Toal

This conference paper is available at ARROW@TU Dublin: https://arrow.tudublin.ie/cieocon2/4 
2009-01-01

\section{A Single Beam Data Writing Process for Holographic Data Storage}

Suzanne Martin

Dublin Institute of Technology, suzanne.martin@dit.ie

Dennis Bade

Dublin Institute of Technology, dennis.bade@dit.ie

Izabela Naydenova

Dublin Institute of Technology, izabela.naydenova@dit.ie

Vincent Toal

Dublin Institute of Technology, vincent.toal@dit.ie

\section{Recommended Citation}

Martin, S.; Bade, D.; Naydenova, I.; Toal, V.; , "A single beam data writing process for holographic data storage.," Optical Data Storage Topical Meeting, 2009. ODS '09. , vol., no., pp.70-72, 10-13 May 2009 doi: 10.1109/ODS.2009.5031757

This Conference Paper is brought to you for free and open access by the Centre for Industrial and Engineering Optics at ARROW@DIT. It has been accepted for inclusion in Articles by an authorized administrator of ARROW@DIT. For more information, please contact

yvonne.desmond@dit.ie, arrow.admin@dit.ie. 


\title{
A single beam data writing process for holographic data storage.
}

\author{
Suzanne Martin, Dennis Bade, Izabela Naydenova, Vincent Toal \\ Centre for Industrial and Engineering Optics, \\ Dublin Institute of Technology. \\ Dublin, Ireland \\ Suzanne.martin@dit.ie
}

\begin{abstract}
A concept is described that could allow Holographic Data Storage with angular multiplexing using, uniquely, only one data writing beam; pre-recorded 'seed' gratings are illuminated with one writing beam in order to produce a data grating.
\end{abstract}

Keywords-Holographic Data Storage; Photopolymer; Grating,

\section{INTRODUCTION}

In Holographic Data Storage (HDS) systems the storage of data, either in bit or page format, involves the recording of a grating or hologram [1]. Normally this means the use of two coherent laser beams, the splitting and manipulation of which makes the optical head bulky and complex. Efforts are constantly being made to simplify recording. A single beam data writing system would therefore have very considerable advantages in simplifying the data writing system in HDS.

The new concept described here could allow holographic data storage with angular multiplexing using only one data writing beam. In standard systems two beams interfere in the medium in order to create the holographic grating or hologram during the data writing step. For angular multiplexing the angles of incidence of the recording beams must be altered between recordings while maintaining the correct overlap within the medium and retaining interferometric stability. This necessitates a certain degree of complexity in the optical system.

With the concept described here, a series of identical weak holographic gratings are multiplexed into the recording medium at the manufacturing stage, so that at the data writing stage these weak 'seed' gratings can be illuminated with a single beam in order to create new gratings. Diffraction at the 'seed' grating provides the second interfering beam, thereby eliminating the need for two beams at the data writing stage.

In order for this approach to work, the recording material used must respond appropriately to the single data writing beam. It must be capable of producing an altered diffracted (signal) beam, and it must only do so near the Bragg angle of the seed grating (for angular multiplexing).
In many holographic recording materials illumination with a single beam of light (involving no interference pattern) while the material is still photosensitive will erase any existing grating. However, it has been known for many years that the diffraction efficiency of gratings recorded in certain self developing polymer systems can be increased by single beam illumination [2], and we have used our own self-developing photopolymer to demonstrate the concept discussed here. Photorefractive materials can exhibit similar behavior, and although they form transient gratings, the process in our photopolymer is thought to be in some ways analogous to the weak beam/strong beam interactions in photorefractive crystals.

The increase in diffraction efficiency that's observed under single beam illumination in our photopolymer is thought to be due to a new grating formed by the interference between the single writing beam and the 1st order beam generated by diffraction at the pre-recorded weak grating. Since the recording processes are very rapid [3] in these materials, any increase in efficiency of the grating quickly produces a stronger reference (diffracted) beam, reducing the beam ratio and increasing the contrast in the interference pattern. This increase in contrast further enhances the refractive index modulation through the normal recording processes, in turn producing a stronger diffracted beam. In this way, quite weak gratings can 'seed' the growth of relatively high diffraction efficiency gratings. When single beam illumination is perfectly on-Bragg the growth of the new grating is evidenced by an increase in the diffraction efficiency of the original grating.

Our preliminary results support this view by demonstrating the reduced response as the writing beam is moved away from the Bragg angle of the 'seed' grating. Our results also demonstrate the ease with which the 'seed' gratings can be enhanced ( or new gratings created ) with one writing beam. In our photopolymer, gratings of less than $1 \%$ efficiency can be 'enhanced' with a single beam to give diffraction efficiencies many times greater than the original value. The Bragg selectivity of the recording process and the recorded data gratings is also demonstrated. 
Both bit-wise and page-wise recording formats would be possible with this single writing beam approach. In the simplest case of a bit-wise data storage system, the diffraction efficiency of the pre- recorded 'seed' grating is altered using the single data writing beam, so that at read-out, the diffraction efficiency is greater. The enhanced grating represents bit ' 1 ' and an unchanged seed grating represent bit ' 0 '. In a page-wise approach, the single beam used at the data writing stage is spatially modulated to encode a data page. This results in a spatial modulation in the intensity of the diffracted beam.

In either the bit-wise or page-wise data storage system the regular two incident beam holographic recording process is only needed during manufacture, removing the complexity of two-beam recording from the end user's data writing system, while retaining the advantages of angular multiplexing and large storage capacity. The end user then requires a much simpler optical system with only one writing beam to write the data to the 'blank' disk.

\section{RESULTS}

Figure 1 demonstrates the grating enhancement, or diffraction efficiency increase produced in our photopolymer when a pre-recorded grating is illuminated on-Bragg with only one of the original writing beams. A standard two-beam recording of 2 s duration of a grating with spatial frequency 500 lines $/ \mathrm{mm}$ is followed by a $25 \mathrm{~s}$ delay, then single beam exposure starts at $27 \mathrm{~s}$.

In this example the diffraction efficiency is observed to increase from below $2 \%$ to $20 \%$ in the first 70 seconds of single beam exposure. The photopolymer formulation is the standard formulation that we have previously described in [3].

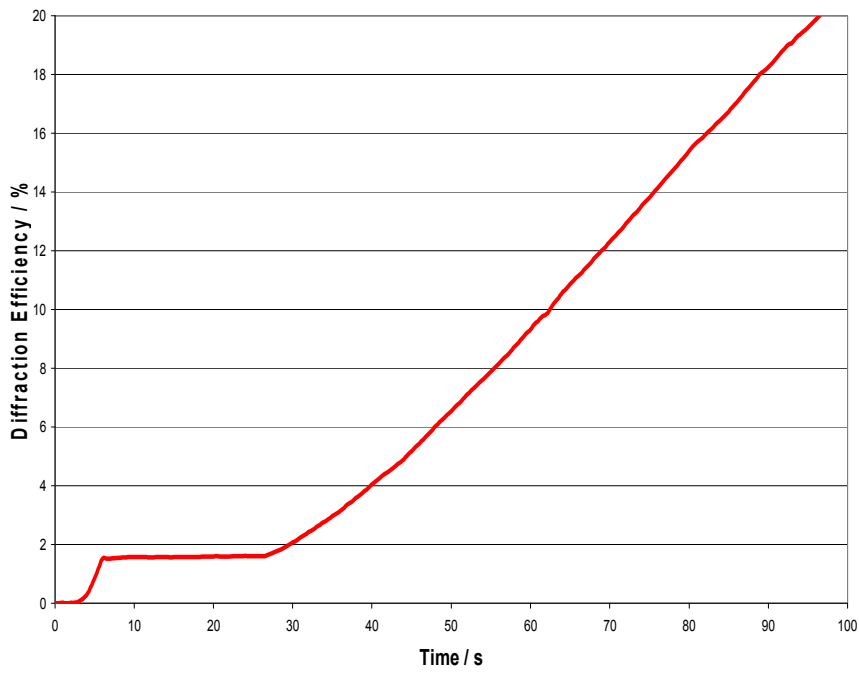

Figure 1. Diffraction efficiency versus time during the single beam illumination process: A standard two-beam holographic recording of $2 \mathrm{~s}$ to form a grating with spatial frequency 500 lines $/ \mathrm{mm}$ is followed by a $25 \mathrm{~s}$ delay, and then exposure to just one of the recording beams.
Figure 1 shows that significant diffraction efficiency increase is possible from exposure to a single on Bragg recording beam. This is a far higher increase than would be necessary to create a strong signal in a data recording system. In a real system it would, of course, be necessary to multiplex a large number of gratings in the same photopolymer layer, so the effect of writing off-Bragg was also studied. A series of gratings were recorded using the single beam exposure method described above, except that the angle of incidence of the single beam was varied slightly on either side of the Bragg angle.

Figure 2 is a graph showing Bragg curves (the variation of diffraction efficiency with angle of incidence of the reading beam) for a series of gratings recorded using a single beam to illuminate a pre-recorded seed grating. The pre-recorded gratings were identical, but in each case the single exposing beam was offset by a different amount from the original writing angle. The Bragg curves shown here were obtained by scanning a reading beam through a range of angles close to the Bragg angle of the original seed grating. The original seed grating diffraction efficiencies were close to $1.3 \%$ in each case and the photopolymer thickness was 135 microns. The spatial frequency of the seed grating was 500lines $/ \mathrm{mm}$.

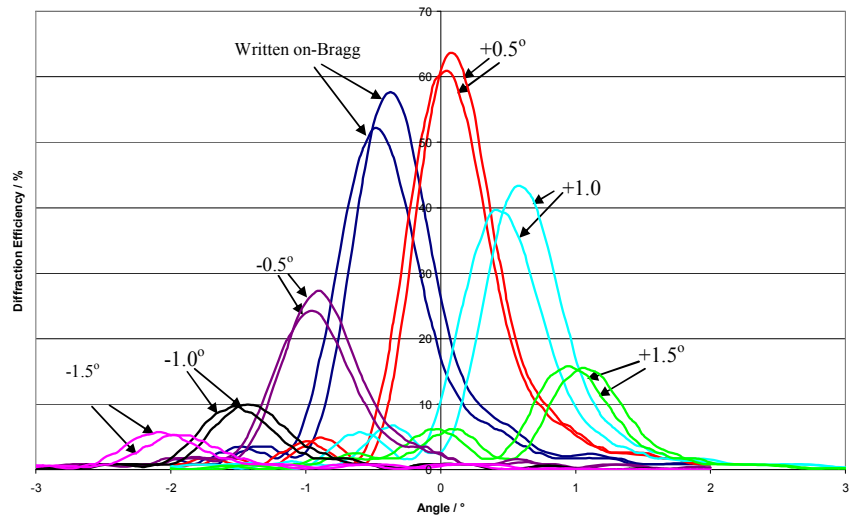

Figure 2. Bragg curves for a series of gratings formed using the single beam process using different angles of incidence of the single writing beam. The gratings were recorded in different photopolymer layers, but are shown here on on one graph for comparison purposes. The arrows indicate the offset (in degrees) from the Bragg angle of the seed grating $\left(0^{\circ}\right)$. There are two identical recordings at each angle.

\section{A number of observations may be made from figure 2:}

(1) As the illuminating beam moves further away from the seed grating's Bragg angle the final diffraction efficiency is less under the same exposure conditions. This is probably due to the reduced coupling between the single writing beam and the pre-recorded grating.

(2) At this spatial frequency and wavelength $(532 \mathrm{~nm})$, at angles more than 1.5 degrees from the Bragg angle there is no new grating of significant efficiency formed. This is important for the design of any multiplexing system based on this process, as a balance will need to be struck between 
minimization of crosstalk, maximization of the storage capacity and the ratio of the number of seed gratings to the number of data gratings.

(3) Despite a slight asymmetry in the Bragg curve, the width of the Bragg curve is only slightly greater in gratings formed through single beam recording compared to that of a regular two-beam grating in the same sample thickness.

(4) The graph shows that there is a possibility of choosing the angular position of the final grating by illuminating slightly off-Bragg.

Finally, it can be observed from Figure 2 that each of the secondary gratings have Bragg curves shifted in the direction of the angular offset of the single beam used to write them. This indicates that there is a tilt in the grating fringes of each secondary grating that depends on the angle of incidence of the writing beam. This relationship between the incident angle of the single writing beam and the position of the Bragg peak for the recorded grating is further evidence for the mechanism of enhanced grating formation proposed above. If the new grating is produced by the interference between the single writing beam and the first order beam produced by the pre-recorded grating, its Bragg angle would be expected to shift linearly with the shift in the writing beam. Figure 3 shows the Bragg peak position plotted against the angle of incidence of the single writing beam. The linearity is strong evidence for the idea that the grating enhancement process depends on the interference between the first and zero order beams. This also has interesting implications for data storage applications because it means that one seed grating can be used to create a data grating at a range of angles near to the seed grating's Bragg angle.

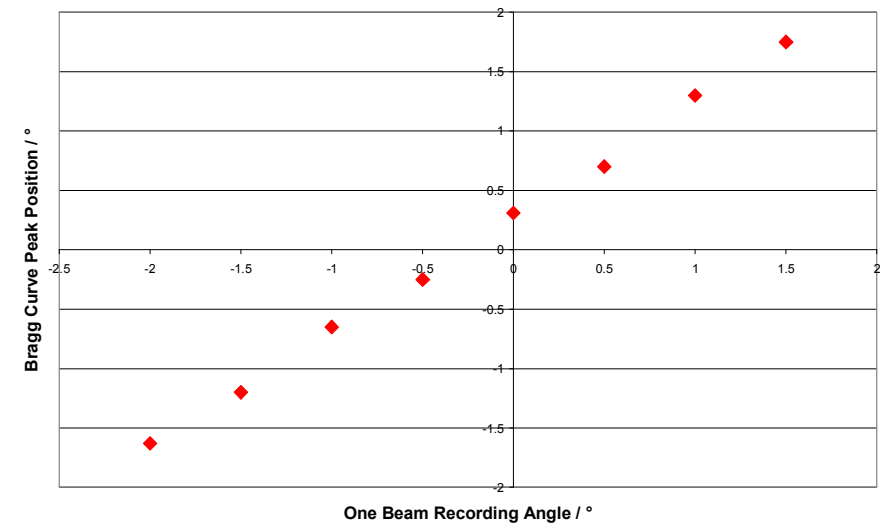

Figure 3. Angular position of the Bragg curve versus single beam angle of incidence (relative to the Bragg angle for the seed grating) from the data shown in Figure 2.

An offset was always observed (approximately 0.5 degrees at this thickness) that we have not yet fully explained, but it may be related to the tilt observed in fringes created when a strong and a weak beam interact in photorefractive materials [4].

Future work will include the demonstration of simple bitwise and page-wise data storage systems with single beam data writing, as well as further investigation into the recording process, and demonstration in other self-developing materials.

\section{CONCLUSIONS}

We have demonstrated that if a weak diffraction grating is recorded in our photopolymer, then a single light beam subsequently interacting with this grating can produce a new grating many times stronger than the original [5]. Depending on the angle of incidence of the single beam the new grating can be superimposed on the original one or be angularly shifted with respect to it. It is proposed that the pre-recorded grating diffracts the light out of the single illuminating beam to produce a weak second beam, which, interfering with the first, creates an interference pattern that the material is capable of recording. The contrast of the pattern is undoubtedly low, but if the single illuminating beam is perfectly on-Bragg, this recording will re-enforce the original grating. In our system such significant diffraction efficiency increase only occurs with when the angle of single beam illumination is at, or very close to, the Bragg angle for the pre-recorded grating. This means that in a data recording system individual gratings will be illuminated and their diffraction efficiency increased while others remain unchanged. Work is underway to prove the concept in data recording systems for bit-wise and page-wise recording.

\section{ACKNOWLEDGMENTS}

The authors would like to acknowledge FOCAS and the School of Physics at DIT for access to facilities and technical support, Hothouse at DIT and Enterprise Ireland for advice and support on Intellectual Property protection and Strand 1 of the Department of Education technological sector research programme for financial support. We are grateful to Dr Gilles Pauliat of the Institut d'Optique, at Orsay in Paris, for interesting discussions on the similarities between our photopolymer system and photorefractives.

\section{REFERENCES}

[1] L. Hesselink; S.S. Orlov and M.C. Bashaw, "Holographic data storage systems" Proceedings of the IEEE, Vol. 92, Issue 8, Aug. 2004, pp. $1231-1280$.

[2] S. Martin, C. A. Feely, J. T. Sheridan, V. Toal, "Applications of a self developing photopolymer material: Holographic interferometry and high efficiency diffractive optical element", Optical Memory and Neural Networks, Vol. 7, No. 2, 1998, pp 79-87.

[3] I. Naydenova, S. Martin, R. Jallapuram, R. Howard and V. Toal, "Investigations of the diffusion processes in self-processing acrylamidebased photopolymer system", Applied Optics, 43 (14), 2004, p 2900.

[4] K. Buse, S. Kamper, F. Physik, J. Frejlich, R. Pankrath and K. H. Ringhofer, "Tilting of holograms in photorefractive $\mathrm{Sr} 0.61 \mathrm{Ba} 0.39 \mathrm{Nb} 2 \mathrm{O} 6$ crystals by self-diffraction" Optics Letters, Vol. 20, No. 21, 1995, pp. 2249-2251.

[5] S. Martin, D. Bade, I. Naydenova, V. Toal, Patent application filed May 2007 